\title{
Modélisation numérique du comportement thermique d'un disque de frein
}

\section{Approche aux conditions limites glissantes}

\author{
Hédi Kchaou* — Mohamed Kaffel** — Fadi Chehadi** \\ Mounir Baccar** \\ * Laboratoire des Systèmes Electromécaniques (LASEM) \\ ** Unité de Dynamique des Fluides Numérique et Phénomènes de Transfert \\ Département de Génie Mécanique, Ecole Nationale d'Ingénieurs de Sfax \\ Route de Sokra, B P $<W>$, 3038 Sfax, Tunisie
}

RÉSUMÉ. La température est un paramètre important dans l'étude et l'interprétation du comportement thermomécanique d'un dispositif de freinage. En effet, l'affaiblissement du coefficient d'adhérence ou le grippage, prenant lieu entre les deux antagonistes patin-disque, dépendent fortement des niveaux de température atteints à la surface de contact, des gradients de températures, et de leurs variations au cours de l'opération de freinage. Dans le présent travail, nous présentons une modélisation numérique tridimensionnelle $d u$ comportement thermique d'un disque de frein ventilé. Le frein faisant l'objet de cette étude est un système à antiblocage 'Anti lock-braking System: ABS'. Afin de tenir compte du chargement thermomécanique mobile au niveau du contact patin-disque, une technique de conditions aux limites glissantes a été développée. Les résultats sont présentés sous forme de cartographies donnant l'évolution des champs de températures dans différents plans $r-\theta$ et $r-z$ en fonction du temps, et ce pour différents scénarios de freinage.

ABSTRACT. Temperature is an important parameter in the design and interpretation of the thermomechanical behaviour of braking systems. The frictional heat generated on the diskpad interface induces high temperature which can cause disk brake fade phenomena, local scoring, thermal cracking and thermo elastic instabilities. In this study we present a thermomechanical modelling of an automotive braking system. For this purpose, a three dimensional simulation thermal model for a ventilated brake-disk of an Anti lock-Braking System (ABS) is conducted in instationary state in different operating conditions. Sliding boundary conditions techniques were developed to consider the effects of the thermomechanical load source (the pad) motion with a decelerated speed. The numerical results were presented in the form of cartographies showing the temperature fields versus time in the $r$ - $\theta$ and $r$-z planes for different braking scenarios.

MOTS-CLÉS: disque de frein, champ de température, chaleur de frottement, conduction instationnaire, condition limite glissante, volumes finis, simulation numérique.

KEYWORDS: disc brake, temperature field, frictional heat, transient conduction, sliding boundary condition, finite volume, numerical simulation.

DOI:10.3166/REMN.17.1057-1075 @ 2008 Lavoisier, Paris 


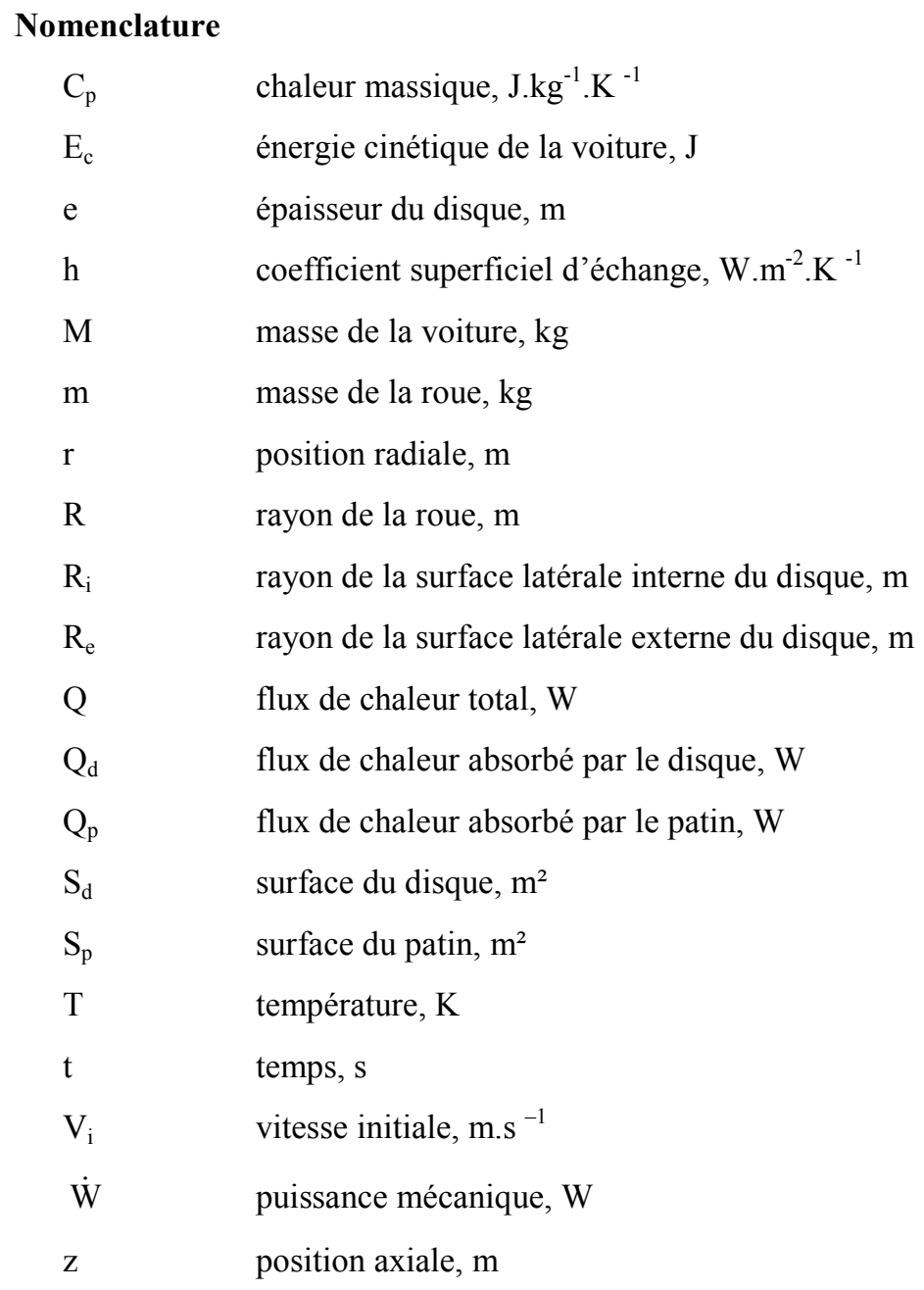

Symboles grecs

$\lambda \quad$ conductivité thermique, $\mathrm{W} \cdot \mathrm{m}^{-1} \cdot \mathrm{K}^{-1}$

$\gamma \quad$ décélération de la voiture, $\mathrm{m} . \mathrm{s}^{-2}$

$\rho \quad$ masse volumique, $\mathrm{kg} \cdot \mathrm{m}^{-3}$

$\xi \quad$ effusivité thermique, $\mathrm{J} \cdot \mathrm{s}^{-1 / 2} \cdot \mathrm{m}^{-2} \cdot \mathrm{K}^{-1}$

$\theta \quad$ position angulaire, $\mathrm{rad}$

$\dot{\theta} \quad$ vitesse angulaire, rad. $^{-1}$

$\ddot{\theta} \quad$ accélération angulaire, $\operatorname{rad} . \mathrm{s}^{-2}$ 


\section{Introduction}

L'analyse thermique du disque de frein est une étape importante pour l'étude et l'interprétation du comportement thermomécanique d'un dispositif de freinage. En effet, l'affaiblissement du coefficient d'adhérence ou le grippage prenant lieu entre les deux antagonistes patin-disque, dépend fortement des niveaux de température atteints à la surface de contact, des gradients de températures, et de leurs variations au cours de l'opération de freinage. Le système de freinage étudié est du type ABS qui assure lors de l'opération de freinage un asservissement de vitesse de chaque roue afin de garder un taux de glissement de la roue par rapport à la route, compris entre $8 \%$ et $30 \%$ (Bosch, 2002 ; Wu et al., 2003). Le chargement est représenté par un flux de chaleur calculé à partir de la perte d'énergie cinétique du véhicule, et dont la distribution spatiale est évaluée à partir du profil des pressions sur le disque conformément à un scénario de freinage donné.

Cette chaleur produite peut provoquer une élévation excessive de température conduisant à des effets indésirables, tels que la fragilisation du disque, l'affaiblissement du coefficient d'adhérence ou le grippage. Simultanément, l'élévation de température ainsi que les forts gradients de température en espace et temps modifient les caractéristiques thermiques et mécaniques et accélèrent l'endommagement du disque sous l'effet de la pression exercée par les patins contre le disque (Lee et al., 1969). Ainsi, la connaissance du champ de température et son évolution temporelle permet de préciser les conditions de freinage les plus endommageantes du disque. Cette connaissance ne peut être fournie que par voie de simulation numérique, car il est difficile de connaître les températures à l'intérieur du disque par les moyens d'investigation expérimentale conventionnels.

Ainsi, la détermination numérique du champ de température a permis à Altaweel et al. (1999) de localiser les zones chaudes sur la surface du disque où la température peut dépasser $450^{\circ} \mathrm{C}$ et atteindre même $800^{\circ} \mathrm{C}$ pour un freinage d'urgence. Dans ces conditions extrêmes de freinage, l'analyse microscopique de l'état de surface du disque a montré l'existence de fissures amorcées.

Cependant, parmi les travaux numériques antérieurs (Lee et al., 1969 ; Altaweel et al., 1999 ; Floquet et al., 1996 ; Laraqi et al., 2001 ; Gao et al., 2002 ; Roussette et al., 2003 ; Nguyen et al., 2002, 2004 ; Choi et al., 2004), rares sont les travaux qui tiennent compte du déplacement angulaire de la zone de frottement patin-disque, siège d'un flux de chaleur dissipatif variable ou discontinu. Parmi ces rares travaux, on peut citer ceux de Floquet et Dubourg (1996), qui ont développé un modèle numérique en vue de la simulation du comportement thermomécanique d'un disque de frein ventilé. La méthode développée consiste à subdiviser le système de freinage en une partie fixe liée aux patins et une partie mobile liée au disque dans son mouvement de rotation. A son tour le disque est subdivisé en deux domaines qui se présentent sous forme de deux couches cylindriques adjacentes aux patins et un troisième domaine correspondant à la partie centrale contenant les cannelures de ventilation et le moyeu. L'interaction entre les parties fixe et mobile est prise en compte en imposant un 
premier couplage au niveau de la surface de contact patin-disque et un deuxième couplage interne entre les couches cylindriques et la partie centrale du disque. Ensuite, le calcul des températures dans la partie centrale, contenant un seul canal de ventilation, est entrepris par la méthode des éléments finis. Simultanément, le calcul de la distribution des températures dans la partie superficielle du disque est entrepris par une technique numérique hybride associant la technique de Transformée de Fourier et la méthode des Eléments Finis. Ce schéma de résolution hybride permet de tenir compte de la variation angulaire de la température à la surface du disque.

La difficulté majeure que présente le travail de Floquet et Dubourg (1996) réside dans la réactualisation, en fonction du nombre de Peclet, de l'interface interne séparant les domaines de calcul liés au disque et au niveau de laquelle on peut admettre une symétrie de révolution.

Dans le présent travail, en vue de la prédiction du champ de température dans un disque de frein de type ABS, nous avons développé un code de calcul spécifique intégrant une méthode numérique tenant compte de la variabilité spatio-temporelle du chargement thermique du disque. Cette méthode, dite de conditions limites glissantes permet de :

- tenir compte de la variation instantanée de la position relative du patin par rapport au disque, par synchronisation avec le retardement de la vitesse au cours du freinage,

- mettre fin à une situation de risque de blocage en supprimant momentanément le contact patin-disque, et ce en fonction du scénario de freinage imaginé,

- tenir compte de la variation du flux de chaleur dissipé par le contact patindisque au cours d'un scénario de freinage donné.

L'objectif de ce travail consiste à apporter une connaissance finalisée portant sur la répartition des températures dans un disque d'un système de freinage de type $\mathrm{ABS}$, en réponse à différents scénarios de freinage caractérisés par les différents paramètres se rapportant au contact roue-chaussée. Une telle analyse permet de déterminer l'histoire thermique transitoire $\mathrm{du}$ disque pour une meilleure compréhension de la réponse mécanique de la structure des organes de freinage.

\section{Formulation du problème}

Le dispositif étudié est un disque de frein de type ABS à canaux de ventilation (figure 1). Les propriétés thermophysiques du disque sont déterminées à une température moyenne de $200^{\circ} \mathrm{C}$. Les caractéristiques géométriques ainsi que les propriétés thermophysiques des deux antagonistes patin-disque sont récapitulées dans les tableaux 1 et 2 . Par ailleurs, nous supposons que le coefficient d'adhérence reste constant pendant l'opération de freinage et que le flux de dégradation d'énergie mécanique en chaleur est uniformément réparti sur le secteur de contact patin-disque. 


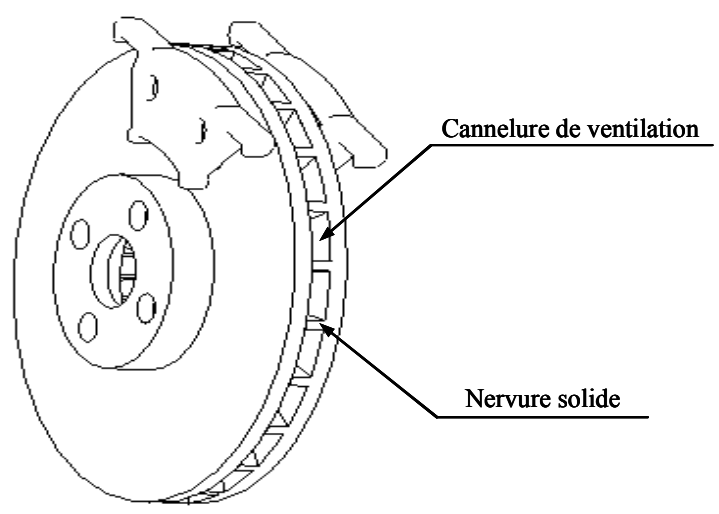

Figure 1. Dispositif étudié

\begin{tabular}{|l|l|}
\hline Rayon intérieur du disque & $\mathrm{R}_{\mathrm{i}}=45 \mathrm{~mm}$ \\
\hline Rayon extérieur du disque & $\mathrm{R}_{\mathrm{e}}=125 \mathrm{~mm}$ \\
\hline Epaisseur du disque & $\mathrm{e}=25 \mathrm{~mm}$ \\
\hline Rayon intérieur du patin & $\mathrm{R}_{\mathrm{ip}}=83 \mathrm{~mm}$ \\
\hline Rayon extérieur du patin & $\mathrm{R}_{\mathrm{ep}}=122 \mathrm{~mm}$ \\
\hline Surface du patin & $\mathrm{S}_{\mathrm{p}}=2384 \mathrm{~mm}^{2}$ \\
\hline
\end{tabular}

Tableau 1. Données géométriques du système de freinage

\begin{tabular}{|c|c|c|}
\hline Propriétés thermophysiques & Disque & Patin \\
\hline Masse volumique & $\rho_{\mathrm{d}}=7100 \mathrm{~kg} \mathrm{~m}^{-3}$ & $\rho_{\mathrm{p}}=1400 \mathrm{~kg} \mathrm{~m}^{-3}$ \\
\hline Chaleur massique & $\mathrm{C}_{\mathrm{d}}=452 \mathrm{~J} \mathrm{~kg}^{-1} \mathrm{~K}^{-1}$ & $\mathrm{C}_{\mathrm{p}}=1000 \mathrm{~J} \mathrm{~kg}^{-1} \mathrm{~K}^{-1}$ \\
\hline Conductivité thermique & $\lambda_{\mathrm{d}}=57 \mathrm{~W} \mathrm{~m}^{-1} \mathrm{~K}^{-1}$ & $\lambda_{\mathrm{p}}=5 \mathrm{~W} \mathrm{~m}^{-1} \mathrm{~K}^{-1}$ \\
\hline
\end{tabular}

Tableau 2. Données physiques des deux antagonistes patin-disque à une température moyenne de $200^{\circ} \mathrm{C}$ (Choi et al., 2004)

L'équation régissant le transfert instationnaire conductif dans le disque de frein, s'écrit dans un système de coordonnées cylindriques tridimensionnelles comme suit :

$$
\rho \mathrm{C}_{\mathrm{p}} \frac{\partial \mathrm{T}}{\partial \mathrm{t}}=\frac{1}{\mathrm{r}} \frac{\partial}{\partial \mathrm{r}}\left(\lambda \mathrm{r} \frac{\partial \mathrm{T}}{\partial \mathrm{r}}\right)+\frac{1}{\mathrm{r}} \frac{\partial}{\partial \theta}\left(\lambda \frac{1}{\mathrm{r}} \frac{\partial \mathrm{T}}{\partial \theta}\right)+\frac{\partial}{\partial \mathrm{z}}\left(\lambda \frac{\partial \mathrm{T}}{\partial \mathrm{z}}\right)
$$


Ou encore sous une forme vectorielle qui se prête à la discrétisation par la méthode des volumes finis :

$$
\frac{\partial \mathrm{T}}{\partial \mathrm{t}}=\operatorname{div}\left(\frac{\lambda}{\rho \mathrm{C}_{\mathrm{p}}} \overrightarrow{\operatorname{grad}} \mathrm{T}\right)
$$

Par raisonnement de symétrie axiale par rapport au plan médian du disque, on s'est limité à l'étude de la moitié du disque. Dans cet article, les résultats sont présentés pour un maillage uniforme de 153 nœuds suivant la direction radiale, 153 nœuds suivant la direction angulaire et 16 nœuds suivant la direction axiale.

\section{Conditions aux limites}

Pour appliquer les conditions aux limites au niveau du contact patin-disque, nous avons convenu de donner un mouvement de rotation aux patins par rapport à un observateur lié au disque. Ainsi, les conditions aux limites au niveau du contact disque-patin, seront animées d'un mouvement de rotation uniformément retardé et glissant dans le sens opposé à celui des roues.

Au cours d'un freinage d'arrêt, l'énergie cinétique du véhicule est convertie en chaleur et travail mécanique :

$$
\frac{\mathrm{dEc}}{\mathrm{dt}}=\dot{\mathrm{W}}+\mathrm{Q}
$$

Le mouvement étant uniformément retardé, la puissance totale de freinage à dissiper n'est pas constante. Elle est maximale au début du freinage et décroît avec la vitesse du véhicule :

$$
\mathrm{Q}=\mathrm{M}\left(\mathrm{V}_{\mathrm{i}}+\gamma \mathrm{t}\right) \gamma-m \mathrm{R}^{2} \ddot{\theta} \dot{\theta}
$$

Cette puissance est absorbée à la fois par le disque et le patin. En supposant que la densité de flux thermique fournie à la garniture du patin $\mathrm{Q}_{p}$ et du disque $\mathrm{Q}_{\mathrm{d}}$ varient dans les mêmes proportions que leurs effusivités respectives $\xi_{\mathrm{p}}$ et $\xi_{\mathrm{d}}$, on peut écrire alors (Roussette et al., 2003 ; Bodovillé., 2001) :

$$
\begin{aligned}
& \mathrm{Q}_{\mathrm{p}}=\frac{\mathrm{S}_{\mathrm{p}} \xi_{\mathrm{p}}}{\mathrm{S}_{\mathrm{p}} \xi_{\mathrm{p}}+\mathrm{S}_{\mathrm{d}} \xi_{\mathrm{d}}} \mathrm{Q} \\
& \mathrm{Q}_{\mathrm{d}}=\mathrm{Q}-\mathrm{Q}_{\mathrm{p}}
\end{aligned}
$$

Ce flux de chaleur est réactualisé à chaque pas de temps en vue de tenir compte de la décélération du mouvement. Parallèlement, et pour tenir compte du déplacement relatif du patin contre la surface du disque (figure 2), nous avons développé une technique dite de condition limite glissante qui permet de réactualiser la position du patin après un laps de temps ' $\mathrm{n} \Delta \mathrm{t}$ ' correspondant à l'équivalent d'un 
pas angulaire $\Delta \theta$. Il s'agit en fait du temps s'écoulant entre deux positions angulaires consécutives du secteur patin-disque. Ainsi, pendant l'intervalle de temps $[\mathrm{t}, \mathrm{t}+\mathrm{n} \Delta \mathrm{t}$, le patin est supposé restant figé dans une même position.

Le pas angulaire étant fixé par le maillage, le pas de temps $\Delta$ t est alors calculé pour chaque position du patin par rapport au disque, par résolution de l'équation du mouvement donnant $\Delta \theta$ en fonction de $\Delta \mathrm{t}$, en prenant délibérément $\mathrm{n}=3$ :

$$
\Delta \theta=\frac{1}{2} \ddot{\theta}(\mathrm{n} \Delta \mathrm{t})^{2}+\dot{\theta} \mathrm{n} \Delta \mathrm{t}
$$

Ainsi, après trois incréments de temps, cette égalité étant vérifiée, on fait alors subir au patin un saut ou un glissement d'un pas angulaire $\Delta \theta$.
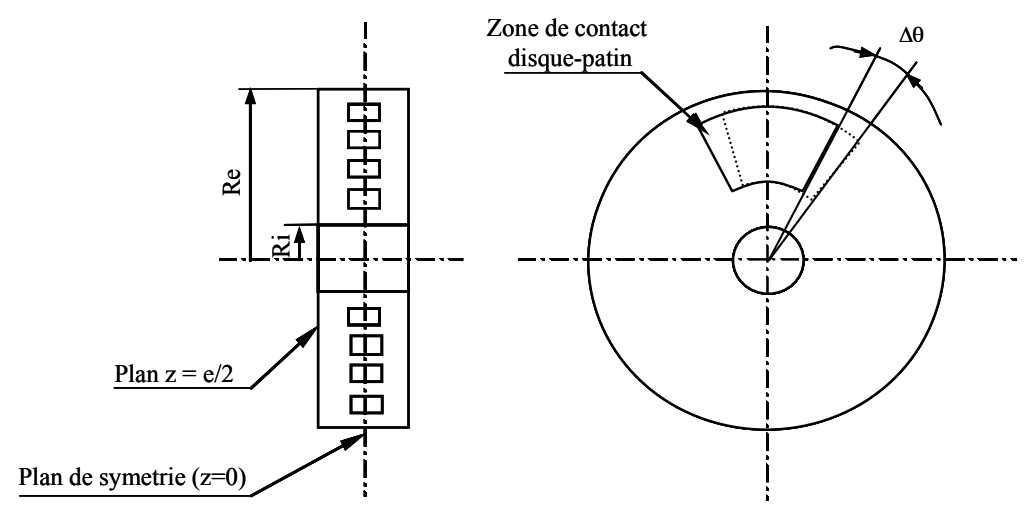

Figure 2. Domaine de calcul et déplacement relatif du patin

Au cours de l'opération de freinage, la chaleur due au frottement patin-disque est partiellement dégagée dans l'air ambiant baignant les surfaces du disque. Ainsi, en dehors de la portion de surface de contact patin-disque, la surface du disque est refroidie par convection, ce qui nous permet d'écrire au niveau du plan $\mathrm{z}=\mathrm{e} / 2$ :

$$
-\lambda \frac{\partial T}{\partial z}=h\left(T_{S}-T_{f}\right)
$$

Le coefficient superficiel d'échange h est égal à $100 \mathrm{~W} \cdot \mathrm{m}^{-2} \cdot \mathrm{K}^{-1}$ (Floquet et al., 1996). $T_{s}$ et $T_{f}$ sont respectivement la température à la surface du disque et celle de l'air en contact qui est supposée constante.

Si l'on considère un scénario de freinage quelconque, tant que le capteur de vitesse ne détecte aucun blocage, le contact patin-disque est maintenu et le flux dissipatif est pris en considération moyennant la technique de condition limite glissante. A l'instant 
du déblocage, les deux antagonistes patin-disque sont libérés, et la portion de surface qui était en contact avec le patin devient exposée à l'air libre pour être refroidie.

Pour tenir compte de la ventilation interne, nous avons supposé que l'air ambiant s'engage dans les cannelures de refroidissement à très grande vitesse, si bien que le coefficient superficiel d'échange entre l'air de refroidissement et les parois internes des canaux, puisse être supposé très grand. Par voie de conséquence, nous avons admis dans notre calcul que la température des parois internes des cannelures est égale à celle de l'air.

Sur les surfaces latérales interne et externe du disque (surfaces cylindriques en $r=R_{i}$ et $r=R_{e}$ ), nous avons imposé des températures constantes égales à la température du milieu ambiant. Au niveau du plan médian coupant une nervure solide du disque de frein $(\mathrm{z}=0)$, nous imposons, par raisonnement de symétrie, une dérivée nulle de la température $:\left.\frac{\partial \mathrm{T}}{\partial \mathrm{z}}\right|_{z=0}=0$.

\section{Résultats et interprétations}

Les véhicules équipés d'un système antiblocage $(\mathrm{ABS})$ présentent un module électronique intégré dans le système de freinage, qui peut détecter un signal indiquant qu'une roue est sur le point de se bloquer. Le module électronique répond en agissant sur un jeu de soupapes montées sur le circuit hydraulique, pour commander les maitres cylindres des roues. Les durées de déblocage et de freinage sont entièrement définies par la centrale électronique du système ABS suivant les informations des capteurs de vitesse sur chaque roue ; la consigne étant une décélération cible définie en fonction de la vitesse instantanée du véhicule. Ces durées dépendent de plusieurs variables, telles que l'état des pneus et de la chaussée, la vitesse initiale du véhicule, le coefficient de frottement au niveau du disque-patin, et la charge du véhicule. Ces différents paramètres influent directement sur le scénario de l'opération de freinage par l'acquisition des vitesses des quatre roues. Instantanément, le module de commande agit en temps réel soit pour freiner soit pour débloquer. Pour reproduire un fonctionnement réel d'un système de freinage $\mathrm{ABS}$, nous allons présenter des visualisations donnant le comportement thermique instationnaire en réponse à trois exemples de profils typiques, en modifiant les différents instants de blocage et de déblocage. Dans tous les scénarios de freinage, la masse du véhicule est fixée à $1200 \mathrm{~kg}$, la vitesse initiale est de $110 \mathrm{~km} / \mathrm{h}$, la décélération cible est de $8 \mathrm{~m} / \mathrm{s}^{2}$ et la température initiale du disque ainsi que celle du milieu ambiant sont égales à $35^{\circ} \mathrm{C}$.

\subsection{Déblocage intervenant entre les instants $t=1 \mathrm{~s}$ et $t=2 \mathrm{~s}$}

La figure 3a représente l'évolution de la pression hydraulique dans le cylindre de roue parallèlement à l'effort de freinage exercé par le patin sur le disque au cours du 
temps. Pendant la première étape (de 0 à $0,5 \mathrm{~s}$ ), on peut constater que l'effort de freinage ainsi que la pression dans le cylindre de roue sont progressifs, et que le patin est en contact permanent avec le disque. Pendant la deuxième étape, s'écoulant entre 0,5 et $1 \mathrm{~s}$, et qui correspond à un risque de blocage de la roue, la pression est maintenue constante par l'isolation de l'huile de freinage dans le cylindre de roue. Si le blocage est sur le point de prendre lieu, l'huile retenue dans le cylindre de roue sera purgée et la pression devient nulle comme on le constate à la troisième étape (de 1 à $2 \mathrm{~s}$ ). Pendant cette dernière étape, le patin n'est plus en contact avec le disque.

Les cartographies donnant l'évolution temporelle des champs de température à la surface du disque sont présentées sur les figures $3 b$ jusqu'à $3 \mathrm{f}$. Tout au début de l'opération de freinage $(\mathrm{t}=0,5 \mathrm{~s})$, la température au niveau de la zone de contact avec le patin atteint déjà une valeur maximale de l'ordre de $200^{\circ} \mathrm{C}$ (figure $3 \mathrm{~b}$ ). Dans le reste de la surface du disque antérieurement balayée par le patin, la température est de l'ordre de $70^{\circ} \mathrm{C}$.

A partir de l'instant $\mathrm{t}=1 \mathrm{~s}$ (figure $3 \mathrm{c}$ ), la roue est sur le point de se bloquer, le contact disque-patin cesse. Le flux de chaleur alimentant le disque s'annule, laissant place à un refroidissement avec l'air ambiant. Sur le secteur préalablement balayé par le patin, les isothermes prennent une forme circonférentielle (figure 3d), et montrent que la tendance est à la baisse de la température et à l'homogénéisation du champ de température.

Le profil donnant la pression dans le cylindre de roue (figure 3a) montre que cette pression reprend sa valeur seuil après 2 secondes et ce, suite à une décélération dépassant la décélération cible de l'opération de freinage. Le patin regagne alors sa position de contact avec le disque, provoquant de nouveau son échauffement (figure 3e) et ramenant le niveau de température dans la zone de frottement à $140^{\circ} \mathrm{C}$ environ. Cette brusque augmentation est cependant moins importante que celle observée en début d'opération de freinage, du fait que le flux de chaleur, dégagé par le contact patin-disque, diminue avec le ralentissement de la voiture.

A la fin de l'opération de freinage, la figure $3 \mathrm{f}$ donnant la répartition des températures sur la surface du disque, montre la présence de taches chaudes qui se succèdent sur la piste de frottement, et ce, à la fréquence de répartition angulaire des nervures sur le disque. A ce stade, la température maximale n'est que de $70^{\circ} \mathrm{C}$.

Pour suivre la variation du chargement thermique du disque, nous avons convenu de reproduire sur la figure $3 \mathrm{~g}$ l'évolution de température du point chaud. Il apparaît qu'à l'instant $\mathrm{t}=1 \mathrm{~s}$, le patin et le disque se séparent et la température chute rapidement de $205^{\circ} \mathrm{C}$ jusqu'à une valeur de $65^{\circ} \mathrm{C}$. Lorsque la roue atteint sa valeur de décélération cible, le patin et le disque sont à nouveau mis en contact à $\mathrm{t}=2 \mathrm{~s}$, ce qui s'en suit d'un sursaut du niveau de température à une valeur de $140^{\circ} \mathrm{C}$. Ensuite, la température diminue progressivement pour atteindre à la fin de l'opération de freinage une valeur de $70^{\circ} \mathrm{C}$. Les fluctuations en dents de scie du profil de température de la figure $3 \mathrm{~g}$, sont dues à l'alternance du frottement au contact patindisque, se produisant à la fréquence de rotation de la roue. 


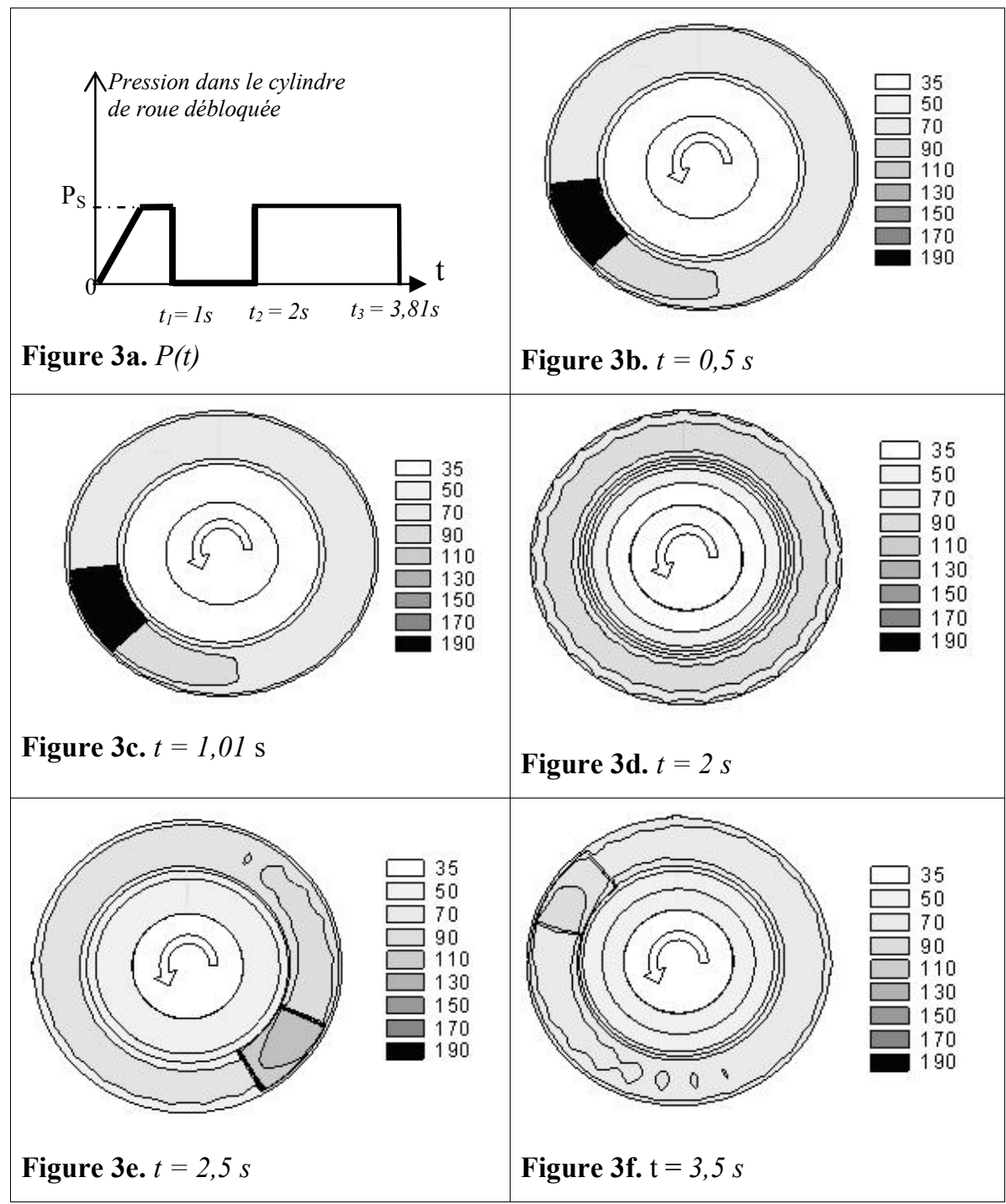

Figure 3. Champs de température à la surface du disque: tendance de blocage entre les instants $t=1$ s et $t=2 \mathrm{~s}$

L'étude de la pénétration thermique à l'intérieur du matériau formant le disque, permet de localiser les forts gradients thermomécaniques favorisant une usure prématurée de la piste de frottement. Les figures $4 \mathrm{a}$ à $4 \mathrm{i}$ et $5 \mathrm{a}$ à $5 \mathrm{i}$, montrent l'évolution temporelle donnant les répartitions des températures dans les plans $\mathrm{r}-\mathrm{Z}$ qui coupent respectivement une cannelure de ventilation et une nervure solide. Ces 
figures montrent bien qu'au début du freinage, les températures les plus élevées sont localisées à la surface de contact avec le patin. Par ailleurs, pendant toutes les étapes de freinage, il apparaît que les gradients de température se concentrent toujours dans le domaine adjacent au patin, même si le contact patin-disque est interrompu comme c'est le cas entre les instants $\mathrm{t}=1 \mathrm{~s}$ et $\mathrm{t}=2 \mathrm{~s}$.

La comparaison des champs de température représentés sur les deux listes de figures montre que les gradients de température sont plus prononcés dans les plans rz passant par les cannelures de ventilation (figures 5a à 5i).

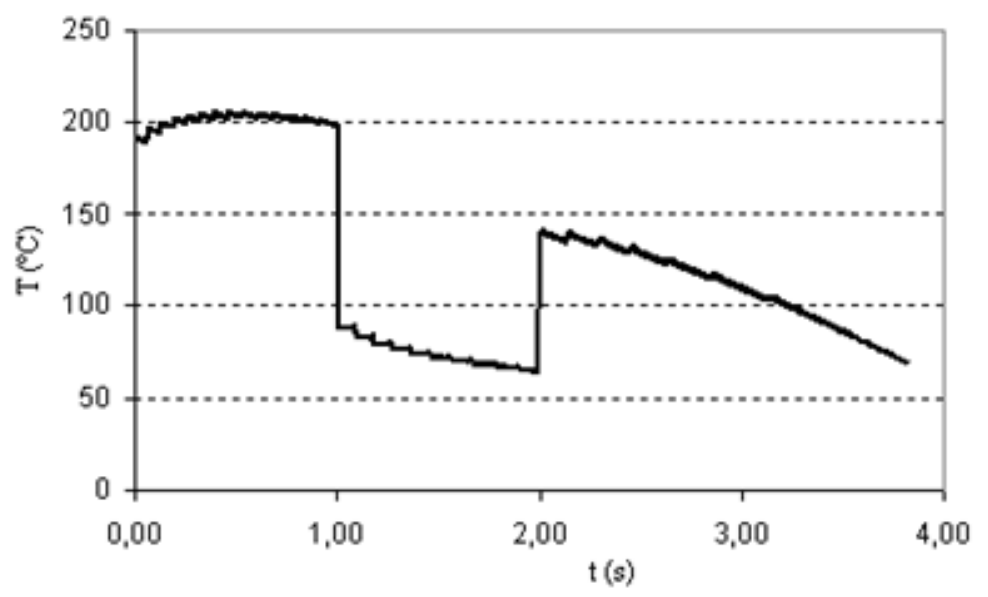

Figure 3g. Evolution de la température dans la zone de contact disque-patin

\subsection{Bref déblocage intervenant entre les instants $t=1 \mathrm{~s}$ et $t=1,5 \mathrm{~s}$}

Dans le présent scénario de freinage, on imagine qu'il suffit de débloquer la roue momentanément pour assurer un freinage efficace qui dure 3,81 s. La vitesse initiale étant de $110 \mathrm{~km} / \mathrm{h}$, le profil de la pression hydraulique dans le cylindre de roue (figure 6a) montre une brève interruption de l'effort de freinage entre les instants $\mathrm{t}=1 \mathrm{~s}$ et $\mathrm{t}=1,5 \mathrm{~s}$. Ce scénario peut survenir par exemple, dans le cas d'un glissement de l'une des roues sur des cailloux ou sur une tache huileuse.

L'évolution de la distribution des températures à la surface du disque est illustrée sur les figures 6-b à 6f. La température maximale atteinte juste avant la tendance au blocage $(\mathrm{t}=1 \mathrm{~s})$, est de l'ordre de $205^{\circ} \mathrm{C}$ (figure $6 \mathrm{~b}$ ). En dépassant la situation de risque de glissement roue-chaussée, le contact disque-patin se rétablit à l'instant $\mathrm{t}=1,5 \mathrm{~s}$, et la température du disque rebondit à nouveau à une valeur de $165^{\circ} \mathrm{C}$ environ (figure 6d). Pour le reste de l'opération de freinage, le véhicule est sensiblement ralenti, et la température maximale à la surface du disque décroît pour atteindre $70^{\circ} \mathrm{C}$ en fin de l'opération de freinage (figure $6 \mathrm{f}$ ). Cette évolution est 
fidèlement reproduite par la figure $6 \mathrm{~g}$, où l'on peut suivre la variation de la température du point chaud du disque, en fonction du temps.

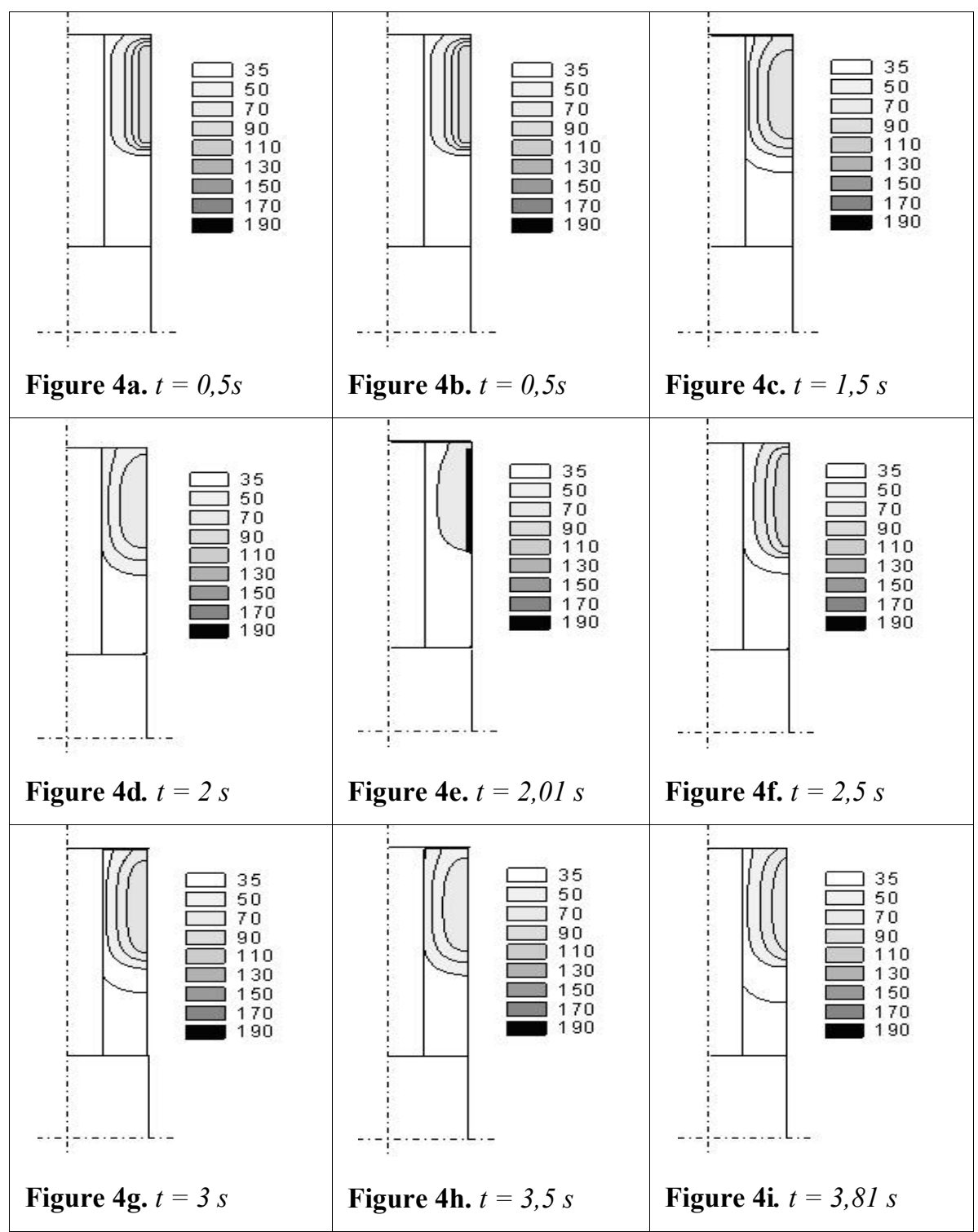

Figure 4. Distribution des températures dans le plan (r,z) passant par une cannelure de ventilation 


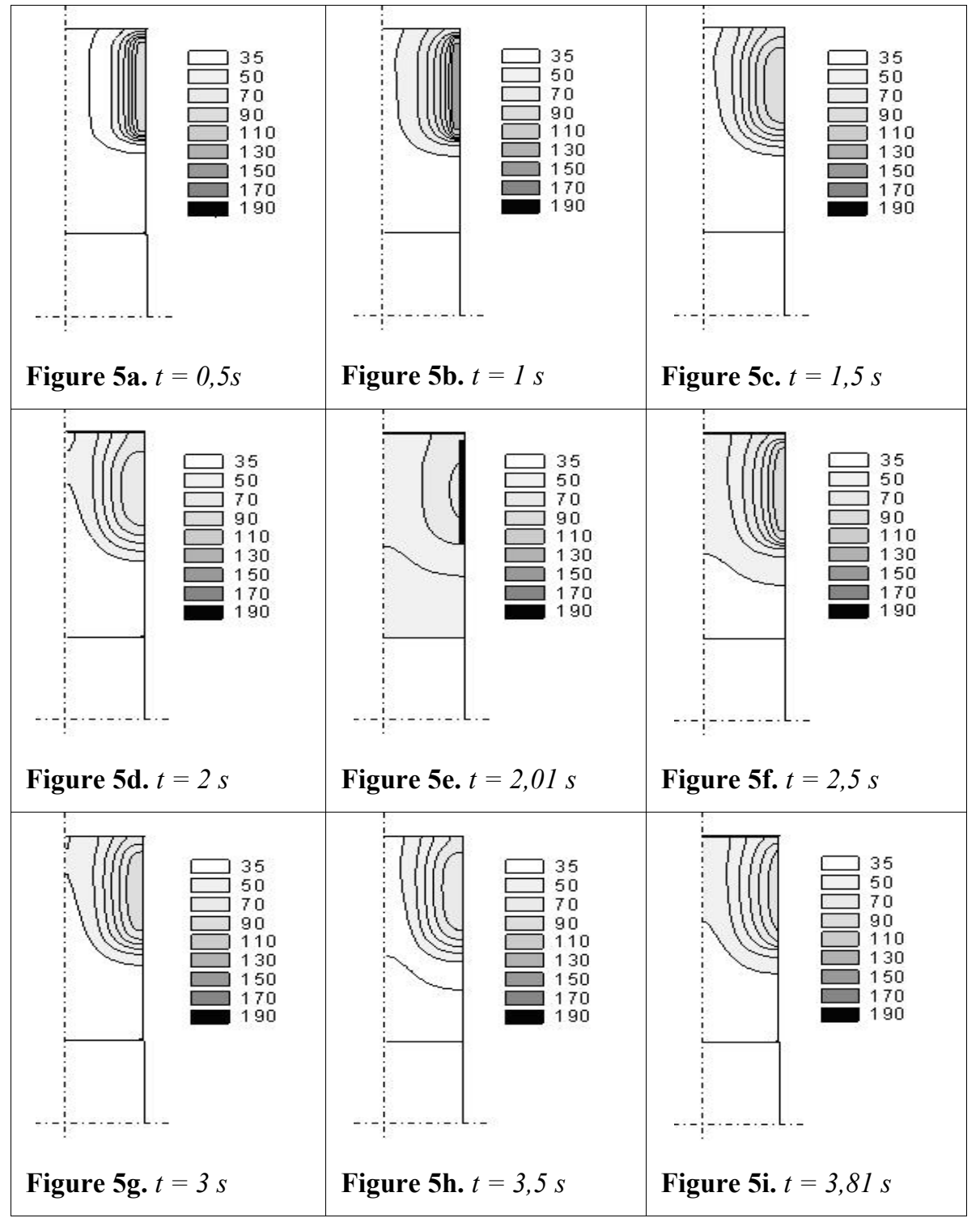

Figure 5. Distribution des températures dans le plan $(r, z)$ passant par une nervure solide 


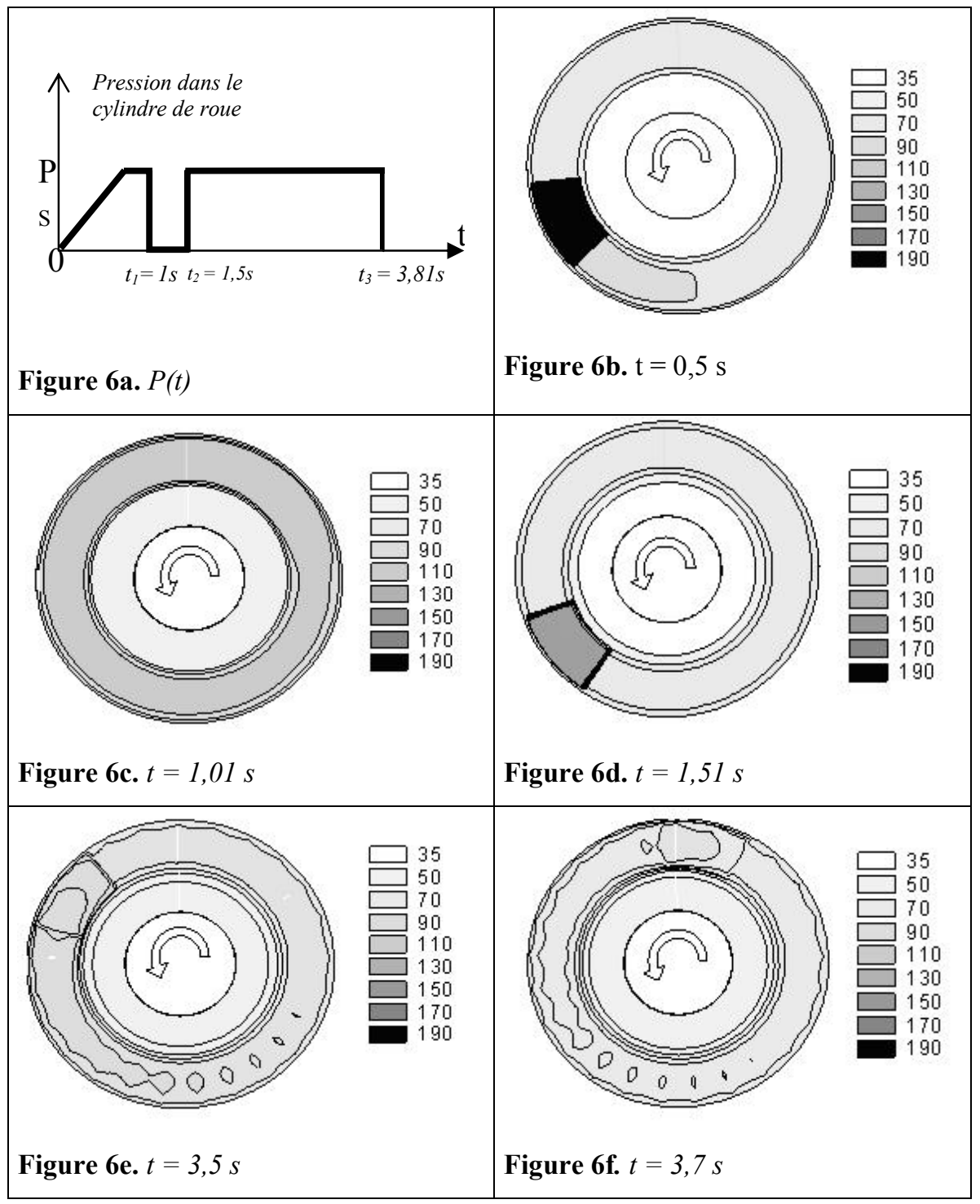

Figure 6. Champs de température à la surface du disque tendance de blocage de l'instant $t=1 \mathrm{~s}$, à $t=1,5 \mathrm{~s}$ 


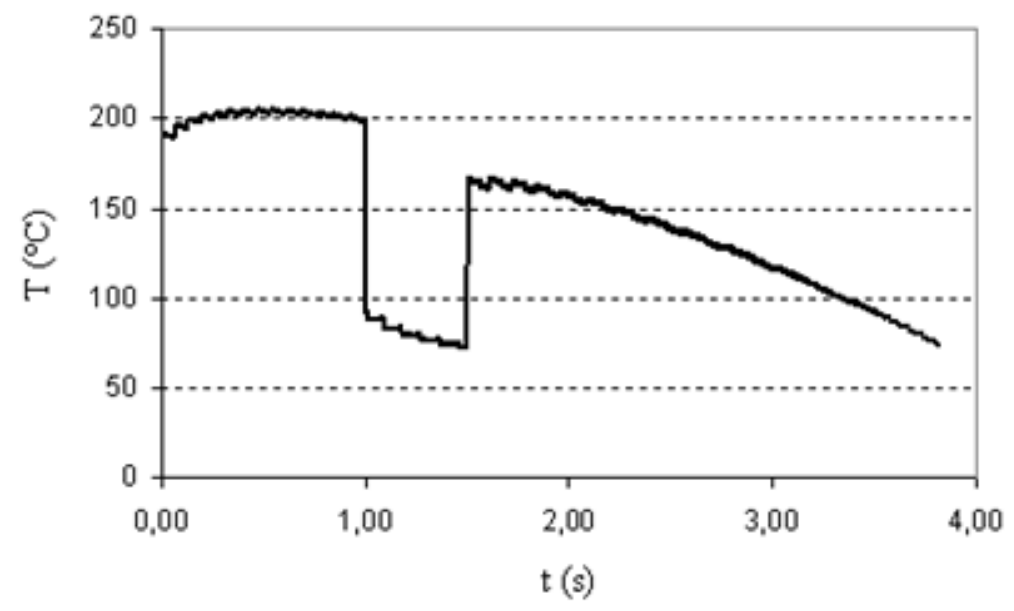

Figure 6g. Evolution de la température dans la zone de contact disque-patin

\subsection{Comportement de deux roues soumises à des conditions de freinage différentes}

Lors d'un freinage d'un véhicule, il peut arriver que deux roues voisines ne se trouvent pas dans les mêmes conditions d'adhérence de leur pneu avec la chaussée : une roue roulant sur une route goudronnée, l'autre sur le bas de coté, par exemple. En cas de freinage avec un système classique, cette situation provoque instantanément la perte de la maîtrise du véhicule et peut causer un dérapage.

Pour un véhicule équipé d'un système $\mathrm{ABS}$, le dérapage n'aura pas lieu, car le système agit en temps réel pour assurer un freinage suivant une décélération optimale, et ce simultanément pour toutes les roues. Ainsi, le comportement thermique des disques des roues n'est pas forcément identique, car ces derniers ne subissent pas le même effort de freinage (figures $7 \mathrm{a}$ et $8 \mathrm{a}$ par exemple).

Les figures $7 \mathrm{~b}$ à $7 \mathrm{f}$ et $8 \mathrm{~b}$ à $8 \mathrm{f}$, mettent en regard l'évolution temporelle des champs de température dans les disques des deux roues voisines soumises à des conditions de freinage différentes.

Il apparaît que lorsque le patin lâche le disque d'une roue qui a tendance à se bloquer (figures $7 \mathrm{c}$ et $7 \mathrm{~d}$ ), la dissipation de l'énergie cinétique du véhicule sera répartie sur les disques des trois autres roues, qui subissent alors un chargement thermique plus important, conduisant à une nette augmentation de la température au contact disque-patin (figure 8c). 


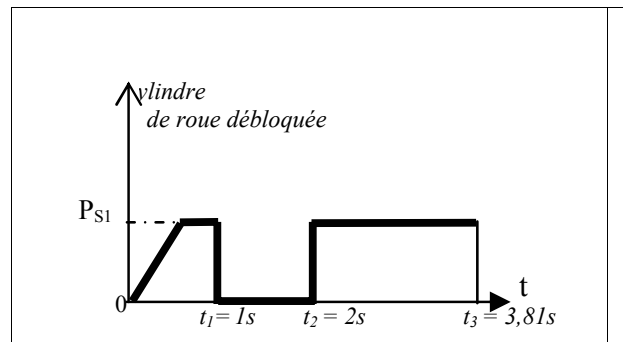

Figure 7a. $P(t)$
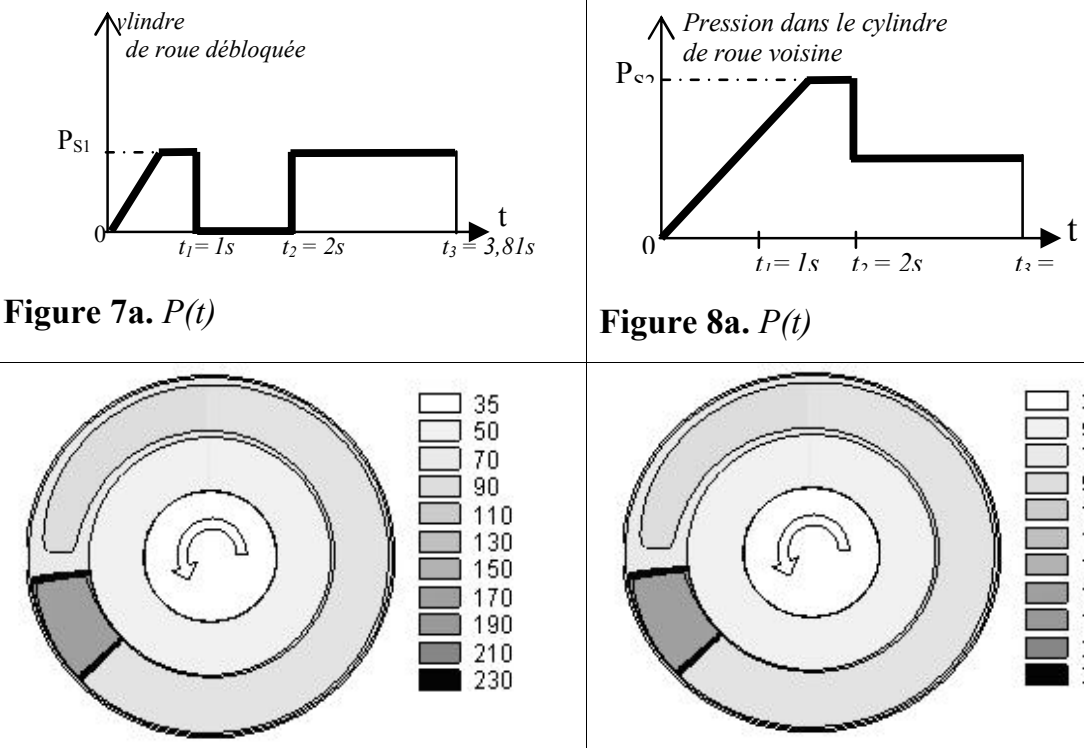

Figure 8a. $P(t)$

Figure 7b. $t=0,5 \mathrm{~s}$

Figure 8b. $t=0,5 s$
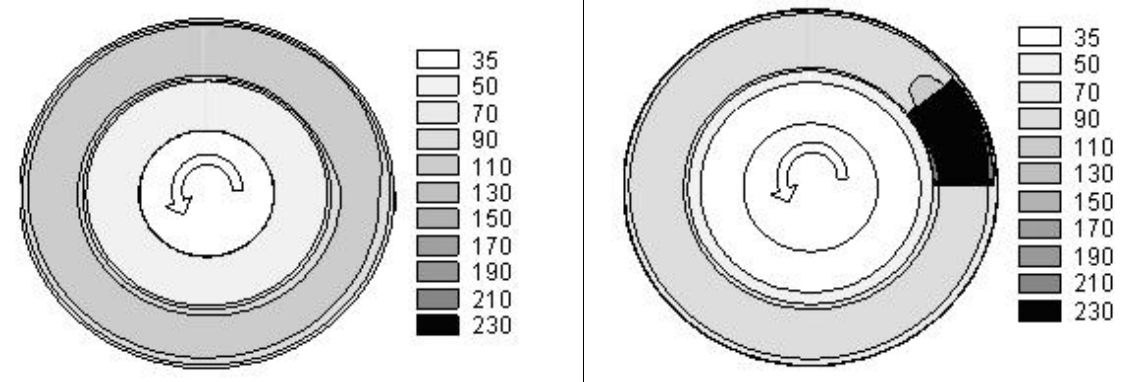

Figure 7c. $t=1,01 \mathrm{~s}$

Figure 8c. $t=1,01 \mathrm{~s}$
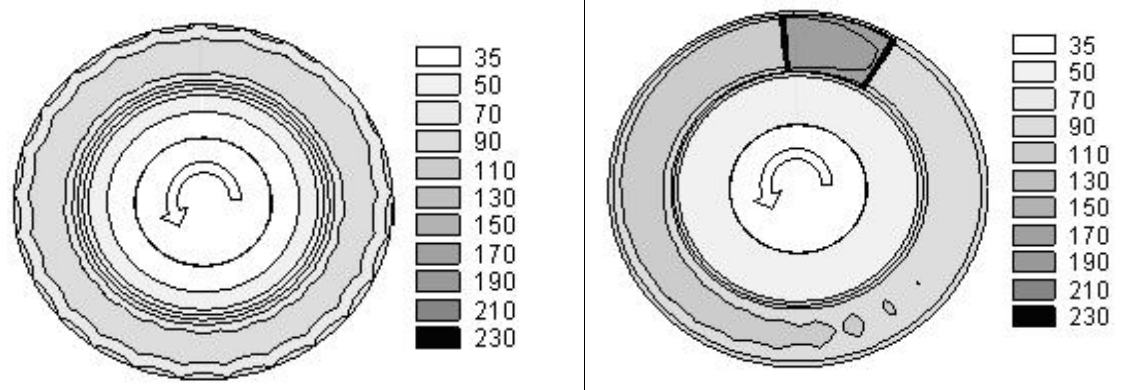

Figure 7d. $t=2 s$

Figure 8d. $t=2 s$ 


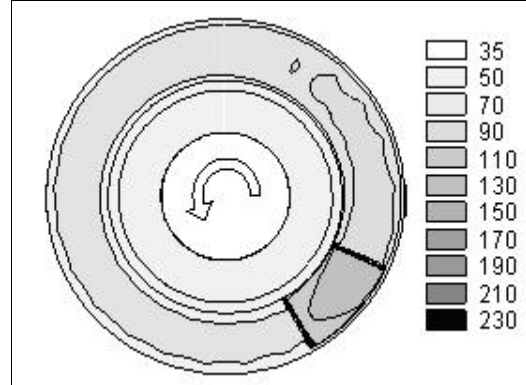

Figure 7e. $t=2,5 \mathrm{~s}$

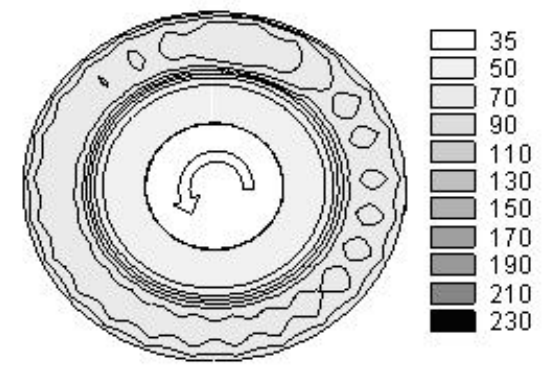

Figure 7f. $t=3,81 \mathrm{~s}$ (arrêt)

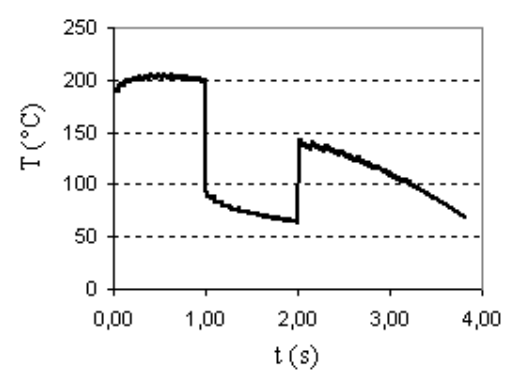

Figure 7g. Evolution de la température dans la zone de contact disque-patin

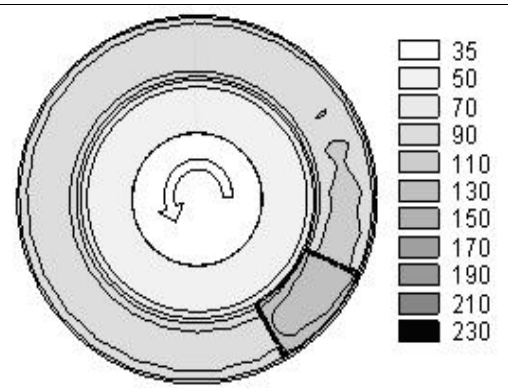

Figure 8e. $t=2,5 \mathrm{~s}$

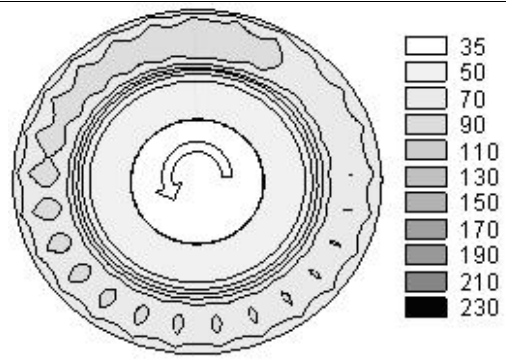

Figure 8f. $t=3,81 \mathrm{~s}$ (arrêt)

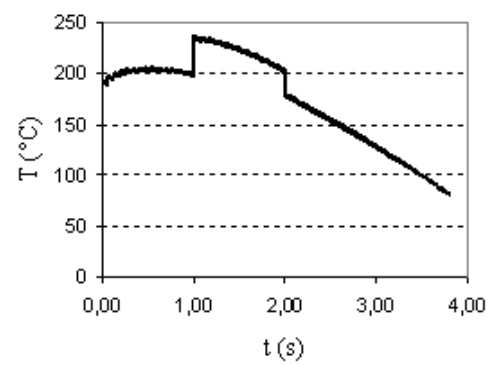

Figure 8g. Evolution de la température dans la zone de contact disque-patin

Figures 7 et 8. Comportement de deux roues soumises à des conditions de freinage différentes

Cette tendance est confirmée par les figures $7 \mathrm{~g}$ et $8 \mathrm{~g}$ qui représentent l'évolution temporelle des températures des points chauds des deux disques soumis au scénario de freinage reproduit par les profils de pression des figures 7a et 8a. Il apparait qu'au moment où la température du point chaud du disque de la roue débloquée chute brutalement jusqu'à $90^{\circ} \mathrm{C}$ environ (figure $7 \mathrm{~g}$ ), car se trouvant en contact avec l'air ambiant, en revanche, le point chaud du disque de l'autre roue connaît une brusque augmentation pour atteindre environ $240^{\circ} \mathrm{C}$ (figure $8 \mathrm{~g}$ ). 
Au terme de l'opération de freinage (figures $7 \mathrm{f}$ et $8 \mathrm{f}$ ), on distingue le défilement de taches chaudes sur la piste de frottement et ce, à la fréquence de répartition angulaire des nervures. L'apparition de ces taches est due à la restitution de la chaleur accumulée au niveau des nervures.

\section{Conclusion}

Dans le présent travail, nous avons présenté une contribution numérique à l'étude du comportement thermique d'un disque de frein de type ABS. A cet effet, nous avons reproduit trois scénarios de freinage en rapport avec des conditions arbitraires de contact roue-chaussée. Pour ce faire, nous avons imaginé différents profils de pression hydraulique dans le cylindre de roue, et calculé la répartition instationnaire des températures dans le disque, en synchronisation avec l'évolution temporelle du profil de pression envisagée.

Sur le plan numérique, nous avons développé une technique, dite de condition limites glissantes, qui permet de réactualiser la position du patin par rapport à un point fixe du disque, en synchronisant l'évolution temporelle et la variation angulaire des températures. Ainsi, en tenant compte de la décélération, on fait déplacer le secteur de contact patin-disque d'un pas angulaire après un nombre déterminé de pas de temps. Le chargement thermique est représenté par un flux de chaleur calculé à partir de la perte d'énergie cinétique du véhicule.

Quel que soit le scénario de freinage, il apparaît que les hautes températures se situent dans la piste de frottement du disque et se concentrent sur le contour des patins. A ce niveau, les gradients de température se montrent aussi très importants. Les contraintes thermiques induites influent sur la réponse mécanique du disque et les dilatations des pièces frottantes modifient la nature du contact. Ceci laisse à présager que des dommages peuvent s'amorcer dans le disque. Parallèlement, ce phénomène peut contribuer à une nette diminution du coefficient de frottement entre les deux antagonistes patin-disque.

Par ailleurs, les résultats donnant les distributions de température à la surface du disque montrent que le niveau de température le plus élevé est atteint juste avant un éventuel blocage. En dépassant la situation de risque de blocage, le contact disquepatin reprend et la température du disque augmente de nouveau très rapidement. Vers la fin de l'opération de freinage, le véhicule étant ralenti, les températures de surface du disque diminuent progressivement jusqu'à l'arrêt total. Ce comportement est vérifié en reproduisant l'évolution temporelle de la température du point chaud de la surface du disque. Il apparaît que le profil de température maximale est marqué par une évolution en dents de scie, à la fréquence de rotation du disque par rapport au patin. 


\section{Bibliographie}

Altaweel B., Pommier S., Priol C., Biausser H., Etude de la fatigue thermique d'une roue de frein, Extrait du rapport d'activité du laboratoire MSS-Mat, 1998-1999.

Bodovillé G., Modélisation thermomécanique de disque de frein de TGV et simulation à échelle réduite, Thèse de doctorat, Université de Lille, 2001.

Bosch R., Système de freinages conventionnels et électroniques, DeltaPress, France, 2002.

Choi J.-H., Lee I., "Finite element analysis of transient thermoelastic behaviors in disk brake", Wear, 257, 2004, p. 47-58.

Floquet A., Dubourg M., "Realistic braking operation simulation of ventilated disk brakes", ASME J. of Tribology, 1996, p. 466-472.

Gao C.-H., Lin X.-Z., "Transient temperature field analysis of a brake in a non-axisymetric three-dimensional model”, J. of Materials Processing Technology, 129, 2002, p. 513-517.

Laraqi N., Bauzin J. G., Alilat N., «Calcul analytique 3D d'un disque de frein », Congrès Français de Thermique, SFT 2001, Nantes, France, 2001, p. 701-706.

Lee K., Barber J.-R., "Thermoelastic instabilities in the sliding of conforming solids", Proceedings of Royal Society, London, 1969, vol. A312, p. 381-394.

Nguyen-Tajan, Maitournam M. et Maestro L., "On a new computational method for the simulation of periodic structures subjected to moving loads: application to vented brake discs", Revue européenne des éléments finis, vol. 13, 2004, p. 627-636.

Nguyen-Tajan, Maitournam M. et Thomas J., «Une méthode de calcul de structures soumises à des chargements mobiles. Application au freinage automobile », Revue européenne des éléments finis, vol. 1, 2002, p. 1-15.

Roussette O., Desplanques Y., Degallaix G., «Représentativité thermique d'essais tribologiques à échelle réduite ", Comptes Rendus Mécanique, 331, n 5, 2003, p. 343-349.

Wu M.-C., Shih M.-C., "Simulated and experimental study of hydraulic anti-lock braking system using sliding-mode PWM control”, Mechatronics, 13, 2003, p. 331-351.

Article reçu le 21 février 2007

Accepté après révisions le 7 août 2008 
